\title{
Assessment of Reinjection Numbers and Intervals for Diabetic Macular Edema Patients Who Received Dexamethasone Intravitreal Implants in Germany and Switzerland
}

\author{
Albert J Augustin (D) \\ Matthias D Becker $\mathbb{D}^{2,3}$ \\ Katja Hatz iD ${ }^{4}$ \\ Hakan Kaymak (iD ${ }^{5}$ \\ Andrew Shirlaw 6 \\ 'Eye Care Clinic, Karlsruhe, Germany; \\ ${ }^{2}$ Department of Ophthalmology, Zurich \\ City Hospital, Zurich, Switzerland; \\ ${ }^{3}$ Department of Ophthalmology, \\ University of Heidelberg, Heidelberg, \\ Germany; ${ }^{4}$ Vista Klinik Binningen, \\ Switzerland and Faculty of Medicine, \\ University of Basel, Basel, Switzerland; \\ ${ }^{5} \mathrm{MVZ}$ Macula and Retina Center Breyer, \\ Kaymak, Klabe, Duesseldorf, Germany; \\ ${ }^{6}$ Allergan, an AbbVie company, Marlow, \\ Buckinghamshire, UK
}

Purpose: The purpose was to assess the number and intervals of dexamethasone intravitreal implant (DEX) reinjections in a real-world setting for the treatment of diabetic macular edema (DME) and to determine the relationship with effectiveness and safety.

Patients and Methods: Data were abstracted from medical records of DME patients in Germany and Switzerland for this retrospective, multicenter, drug utilization study. Bestcorrected visual acuity (BCVA) and central retinal thickness (CRT) changes 7-12 weeks post-injection(s) measured effectiveness. Adverse events (AEs) of special interest were reported.

Results: A total of 141 patients, 108 from Germany and 33 from Switzerland, were assessed. Mean (SD) reinjection interval was 5.7 (4.2) months. Mean baseline BCVA was 61.6 letters, and mean baseline CRT was $413.3 \mu \mathrm{m}$. The mean BCVA and CRT changes at 7 -12 weeks after baseline, reinjection 1,2 , and 3 were $+3.4,+3.7,+3.2$, and -1.4 letters and $-88.3,-81.6,-102.4$, and $-124.1 \mu \mathrm{m}$, respectively. The Spearman correlation between change in BCVA and CRT and DEX reinjection interval was $\mathrm{r}=0.03(P=0.66)$ and $\mathrm{r}=0.07$ $(P=0.38)$, respectively. Only $18 \%$ of patients experienced at least $1 \mathrm{AE}$.

Conclusion: There was no statistically significant correlation between drug effectiveness and reinjection intervals in either country. Although these results are preliminary, they indicate that DEX improves BCVA and CRT in real-world clinical practice.

Keywords: diabetic macular edema, DME, real-world setting, safety, drug effectiveness

\section{Introduction}

Dexamethasone intravitreal implant (DEX) (Ozurdex; Allergan, an AbbVie company, North Chicago, IL, USA) is a biodegradable, solid polymer, drug delivery system that is injected in the vitreous cavity via a single-use applicator with a 22gauge needle. It contains $0.7 \mathrm{mg}$ of the corticosteroid dexamethasone and is designed to provide sustained release of dexamethasone for up to 6 months. ${ }^{1,2}$ It has been approved in the European Union for the treatment of adult patients with visual impairment due to diabetic macular edema (DME) who are pseudophakic or who are considered insufficiently responsive to or unsuitable for non-corticosteroid therapy, and of patients with macular edema following either branch retinal vein occlusion or central retinal vein occlusion, or with inflammation of the posterior segment of the eye presenting as noninfectious uveitis. ${ }^{3}$
Correspondence: Albert J Augustin Moltkestraße 90, Karlsruhe, 76133, Germany

Tel +49 72I-974200I

Email albertjaugustin@googlemail.com 
Diabetic retinopathy is a disease characterized by abnormalities of the retinal blood vessels as a consequence of hyperglycemia. ${ }^{4}$ Patients with diabetic retinopathy can develop DME, an increase of the central retinal thickness (CRT) due to capillary leakage and fluid accumulation. The dangers of DME include the breakdown of the blood-retina barrier and central vision loss. ${ }^{5}$ Since this process also involves inflammatory factors, ${ }^{6}$ the actions of a corticosteroid, such as the one in DEX, can inhibit the progression of DME. ${ }^{7}$

Although previous clinical trials support the safety and efficacy of DEX in treating DME, ${ }^{8-10}$ it is also important to collect data from real-world health care settings. Clinical trials are strictly controlled, and real-world drug utilization does not always reflect those conditions. A realworld assessment of DEX was performed in Canada (CHROME),${ }^{11}$ but did not focus on DME. Another DEX study followed DME patients not previously treated with intravitreal drugs. ${ }^{12}$ The United States (REINFORCE), ${ }^{13}$ Korea, ${ }^{14}$ France, ${ }^{15}$ and Spain ${ }^{16}$ have also conducted realworld effectiveness and safety studies of DEX for the treatment of DME, and results from these studies support that DEX improves best-corrected visual acuity (BCVA) and CRT in real-world clinical practice. Similarly, a retrospective study of DEX using the European DME registry showed that it is safe and effective for the treatment of DME. ${ }^{17}$ It is important to study the real-world effectiveness and safety of DEX in European countries, as well as the populations and practices may differ due to country-specific regulations and reimbursement policies. One treatment aspect of DEX that was not thoroughly assessed in any of these previous studies was the reinjection intervals.

The primary objective of this retrospective chart review study was to assess reinjection intervals of DEX implants in patients with DME in Germany and Switzerland based on real-world data. Other objectives were to assess the relationship between reinjection intervals and drug effectiveness through functional (BCVA) and anatomic (CRT) changes, investigate drug effectiveness in patients that were naïve to anti-vascular endothelial growth factor-(VEGF) therapy prior to their first DEX injection and assess reasons for DEX reinjection. This study will provide real-world data for DEX treatment of DME in European countries that can be used to provide physicians with additional treatment information regarding DEX and can be compared with clinical trial data and other real-world studies.

\section{Methods}

This study was noninterventional, retrospective, and was conducted in compliance with the International Society for Pharmacoepidemiology Good Pharmacoepidemiology Practice (ISPE GPP) guidelines, ${ }^{18}$ the ethical principles arising from the Declaration of Helsinki revised in 2013, ${ }^{19}$ the European Union Good Pharmacovigilance practices (GVP) ${ }^{20}$ and European and National laws in terms of data protection ${ }^{21}$ and all current local regulations, such as Switzerland's Humanforschungsverordnung (HFV) and Humanforschungsgesetz (HFG). Relevant scientific guidelines such as the European Network of Centres for Pharmacoepidemiology and Pharmacovigilance (ENCePP) Guide on Methodological Standards in Pharmacoepidemiology ${ }^{22}$ were considered. Submissions and notifications to the following independent ethics committees were performed in each country as required by local legislation for this type of study: Ethics Committee of Northwestern and Central Switzerland (EKNZ) registration number 2019-00089 (Basel, Switzerland), Ethics Committee of the Faculty of Medicine of the University of Rostock registration number A 2018-0242 (Rostock, Germany), and Ethics Committee of Baden-Württemberg State Medical Association registration number F-2018-095 (Stuttgart, Germany). Due to the retrospective and anonymous nature of data collection for this study, signature of informed consent by patients was not required to abstract data from the patient's chart. This study was registered at www.ClinicalTrials.gov (NCT03889444).

\section{Study Design}

A retrospective, noninterventional, observational, multicenter, drug-utilization, chart review study was conducted in patients implanted with DEX to treat visual impairment due to DME. Medical retina centers treating DME patients in Germany and Switzerland were solicited for participation. This included universities, public hospitals, and praxis. Data were extracted from the medical records of patients in Germany and Switzerland with visual impairment due to DME who fulfill the below mentioned inclusion and exclusion criteria. A minimum of 12 months of data were abstracted from medical charts for each subject, from the date of their first DEX injection up to September 1, 2018. The primary endpoint was to assess reinjection intervals of DEX implants in patients with DME in Germany and Switzerland based on real-world data. Secondary endpoints included assessing the relationship between reinjection intervals and BCVA and CRT responses, drug effectiveness in patients who were naïve 
to anti-VEGF therapy, and reasons for DEX reinjection. Data were anonymized and then entered into an electronic case report form (eCRF) by the investigator or qualified designee. The study eye (SE) was the eye that received the most DEX injections. If both eyes received the same number of injections, the choice of the SE was the investigators' decision. Key relevant data were also collected for the contralateral eye.

\section{Inclusion and Exclusion Criteria}

The inclusion criteria for this study were patients who received at least 2 DEX implants in the SE to treat visual impairment due to DME, adult males or females $(\geq 18$ years of age at the time of the first DEX implant), patients whose first implant occurred after January 1, 2015, and patients who were followed up at the site for at least 12 months after the first DEX implant. Patients who received DEX as part of or during a clinical study were excluded from this study. This was the only exclusion criterion for this retrospective study. The criteria for retreatment were up to the discretion of the physician. The study also focused on a subgroup of patients treated with DEX who were naïve to anti-VEGF treatment. Patients identified for this subgroup met all the inclusion criteria and none of the exclusion criteria listed above, as well as the additional exclusion criteria that the patient received anti-VEGF treatment prior to the first DEX implant injection. Medical charts were screened by the investigative site personnel to identify patients who satisfy all the inclusion and none of the exclusion criteria.

\section{Data Collected}

The parameters collected from patients in this study included: demographics such as patients' age at first DEX implant, gender, and medical and ophthalmic history including chronic medical conditions, DME history, history of glaucoma, and cataract and lens status. Prior and concomitant treatments in the SE such as cataract, glaucoma procedures, laser therapy, other intravitreal injections and medications, clinical examinations, data of the contralateral eye such as DME status, treatments for DME received prior to study period and for each visit, the date of DEX injection, and the reasons for initial and reinjections were also collected. No protocol-specific guidance was given to standardize the reasons for reinjection. They were left to the discretion of the physician and were limited to disease progression, lack of effect of the prior DEX injection, and other (specify). Some physicians specified "relapse" when they chose "other" as the reason for reinjection. Due to the lack of guidance to distinguish between relapse and disease progression, the two were combined for analysis. For the primary objective, the mean (SD) and median reinjection intervals in months were determined overall and individually for Germany and Switzerland. Effectiveness was assessed through changes in functional (BCVA) and anatomic (CRT) efficacy 7-12 weeks post-injection(s). Due to the retrospective nature of the study, all procedures, including BCVA and CRT, were measured using the sites standard procedures, and only data on certain adverse events (AEs) of special interest were collected, primarily based on the summary of product characteristics (SmPC). Information on the causality of events was not collected. Reported AEs of special interest included glaucoma, ocular hypertension, hypotony, cataract or lens opacities, ocular bleeding or hemorrhage, retinal detachment, vitreous detachment, infection versus non-infection-related ocular inflammation, significant vitreous loss, mechanical failure of device and implant misplacement, and implant dislocation. Ocular hypertension was defined as increased ocular pressure without meeting the criteria for glaucoma (nerve damage or vision loss).

\section{Statistical Analysis}

Analyses were primarily descriptive. When appropriate, two-sided 95\% CIs were calculated for specific proportions and means. Statistical analysis was carried out using SAS Studio ${ }^{\circledR}$ version 9.4 on a SAS Grid environment. All values for BCVA analysis were converted into approximate Early Treatment Diabetic Retinopathy Study (ETDRS) letters. Snellen results were converted using this equation: ETDRS $=85+[50 * \log ($ Snellen fraction)]. ${ }^{23}$ The relationship between DEX reinjection interval and BCVA/CRT was assessed by a scatter plot and a linear regression line. The scatter plot had the DEX reinjection intervals in months on the $\mathrm{x}$-axis and either the BCVA change in letters or the CRT change in $\mu \mathrm{m}$ on the $\mathrm{y}$-axis. The Spearman rank correlation coefficient and $P$-value were superimposed on the data of the scatter plot. $t$-Tests were used to calculate $P$ values for change from baseline; values less than 0.05 were considered statistically significant. 


\section{Results}

\section{Patient Demographics}

Of the sites solicited to participate, 11 responded to participate in the study, and 7 sites were initiated. Out of the 7 final sites, 5 were in Germany and 2 were in Switzerland. A total of 141 patients, 108 from Germany and 33 from Switzerland were assessed for this study. The mean (SD) age at the first injection was similar between Germany and Switzerland, with 65.5 (10.2) and 66.3 (9.2) years, respectively. The overall mean was 65.7 (10.0) years. The majority of patients were male in both Germany and Switzerland, $61.1 \%$ and $54.4 \%$, respectively. The mean (SD) time from DME diagnosis to baseline was 23.5 (29.0) and 47.1 (42.0) months in Germany and Switzerland, respectively (Table 1). Mean (SD) overall study duration was 23.3 (8.8) months and was 23.9 (9.0) and 21.3 (8.0) months in Germany and Switzerland, respectively. The severity of diabetic retinopathy ranged from none apparent to proliferative, and most frequently, participants had mild or moderate nonproliferative diabetic

Table I Patient Demographics

\begin{tabular}{|c|c|c|c|}
\hline & Germany & Switzerland & Overall \\
\hline \multicolumn{4}{|c|}{ Age at first DEX injection (years) } \\
\hline $\mathrm{N}$ & 108 & 33 & $14 \mid$ \\
\hline Mean (SD) & $65.5(10.2)$ & $66.3(9.2)$ & $65.7(10.0)$ \\
\hline Min, Max & 31,90 & $4 I, 84$ & 31,90 \\
\hline \multicolumn{4}{|l|}{ Gender $[n(\%)]$} \\
\hline Female & $42(38.9)$ & $15(45.5)$ & $57(40.4)$ \\
\hline Male & $66(61.1)$ & $18(54.5)$ & $84(59.6)$ \\
\hline \multicolumn{4}{|l|}{ Study eye [n (\%)] } \\
\hline OS (Left) & $56(51.9)$ & $13(39.4)$ & $69(48.9)$ \\
\hline OD (Right) & $52(48.1)$ & $20(60.6)$ & $72(51.1)$ \\
\hline \multicolumn{4}{|c|}{ Time from DME diagnosis to baseline } \\
\hline $\mathrm{N}$ & 106 & 31 & 137 \\
\hline Mean (SD) (months) & $23.5(29.0)$ & $47.1(42.0)$ & $28.9(33.7)$ \\
\hline \multicolumn{4}{|c|}{ Anti-VEGF treatment naïve patients } \\
\hline $\mathrm{N}(\%)$ & $44(40.7)$ & I (3.03) & $45(31.9)$ \\
\hline \multicolumn{4}{|c|}{ Number of prior anti-VEGF treatments } \\
\hline $\mathrm{N}$ & 63 & 31 & 94 \\
\hline Mean (SD) & $6.9(7.2)$ & $19.4(16.0)$ & $11.0(12.3)$ \\
\hline Missing & 1 & 1 & 2 \\
\hline \multicolumn{4}{|c|}{ Severity of diabetic retinopathy, n (\%) } \\
\hline None apparent & $15(14.9)$ & $0(0)$ & $15(10.6)$ \\
\hline Mild nonproliferative & $43(42.6)$ & $0(0)$ & $43(30.5)$ \\
\hline Moderate nonproliferative & $30(29.7)$ & $4(12.5)$ & $34(24.1)$ \\
\hline Severe nonproliferative & $8(7.9)$ & $20(62.5)$ & $28(19.9)$ \\
\hline Proliferative & $5(5.0)$ & $8(25.0)$ & $13(9.2)$ \\
\hline Missing & 7 & 1 & 8 \\
\hline \multicolumn{4}{|l|}{ IOP (mmHg): Baseline } \\
\hline Mean $(S D)$ & $15.2(3.7)$ & I5.I (2.5) & $15.2(3.4)$ \\
\hline Median (QI, Q3) & $15.0(13.0,18.0)$ & $15.0(13.0,17.0)$ & $15.0(13.0,17.0)$ \\
\hline Min, Max & 8,26 & $|I, 2|$ & 8,26 \\
\hline Missing & 1 & 2 & 3 \\
\hline Vascular hypertension, n (\%) & $20(15.8)$ & $13(39.4)$ & $33(23.4)$ \\
\hline
\end{tabular}

Abbreviations: Anti-VEGF, anti-vascular endothelial growth factor; DEX, dexamethasone intravitreal implant; DME, diabetic macular edema; IOP, intraocular pressure; max, maximum; min, minimum; OD, right eye; OS, left eye; SD, standard deviation. 
retinopathy. In Germany, 44 patients (40.7\%) were antiVEGF treatment naïve, while in Switzerland, only 1 patient $(3.03 \%)$ was anti-VEGF treatment naïve (Table 1$)$.

\section{Primary Objective: Number of Reinjections and Reinjection Intervals}

Patients received between 1 and 11 DEX reinjections, and the mean (SD) number of reinjections was 2.8 (2.5). In Germany, most patients received 1 or 2 reinjections, while in Switzerland, most received 3 or 4 reinjections (Figure 1). The mean (SD) reinjection interval throughout the entire follow-up period was 5.7 (4.2) months, and the median was 4.6 months. When comparing Germany and Switzerland, Switzerland has shorter reinjection intervals (Table 2).

\section{Effectiveness}

Mean (SD) baseline BCVA was 61.6 (14.9) letters and mean (SD) baseline CRT was 413.3 (128.7) $\mu \mathrm{m}$. Mean (SD) BCVA change from baseline was +3.4 (13.7), +3.7 (10.0), +3.2 (10.9), and -1.4 (12.5) letters 7-12 weeks after the baseline injection, reinjection 1,2 , and $3(P=0.03,0.003$, 0.064, and 0.535, respectively; Figure 2A). Mean (SD) CRT changes were -88.3 (100.2), -81.6 (97.5), -102.4 (117.6), and -124.1 (165.1) $\mu \mathrm{m}$ 7-12 weeks after the baseline injection, reinjection 1,2 , and 3 ( $P \leq 0.001$ for each; Figure $2 \mathrm{~B}$ ). There was no statistically significant correlation between effectiveness measured using BCVA and CRT change from baseline 7-12 weeks after each DEX injection and reinjection interval when observed as a continuous measure. No correlation between the change in BCVA from baseline and DEX reinjection intervals was observed, as the Spearman correlation was $\mathrm{r}=0.03$ with a $P$-value of 0.66 (Figure $3 \mathrm{~A}$ ). The Spearman correlation between the change in CRT from baseline and DEX reinjection intervals was $\mathrm{r}=0.07$ with a $P$-value of 0.38 , also showing no correlation (Figure $3 \mathrm{~B}$ ).

The effectiveness of DEX in patients who were naïve of any anti-VEGF treatment at baseline was compared with those who had prior treatment at baseline. Mean change in BCVA from baseline was higher in the naïve group compared with the anti-VEGF pretreated group after baseline and reinjection 1, although the opposite was seen after reinjection 2. Mean (SD) change in BCVA from baseline was +4.7 (11.8), +4.2 (11.1), and +1.6 (9.6) letters after baseline injection, reinjection 1 , and 2 , respectively, in the treatment-naïve subpopulation. Mean change in BCVA from baseline was $+3.0(14.3),+3.5(9.7)$, and +3.9 (11.5) letters after baseline injection, reinjection 1 , and 2 , respectively, in the subpopulation with prior anti-VEGF treatment (Table 3 ). Mean CRT change from baseline was higher in the antiVEGF pretreated subpopulation compared with the naïve group. In the naïve subpopulation, mean CRT change from baseline was $-74.3(87.9)$ and -63.8 (106.7) $\mu \mathrm{m}$, while in the anti-VEGF pretreated subpopulation, it was -94.5

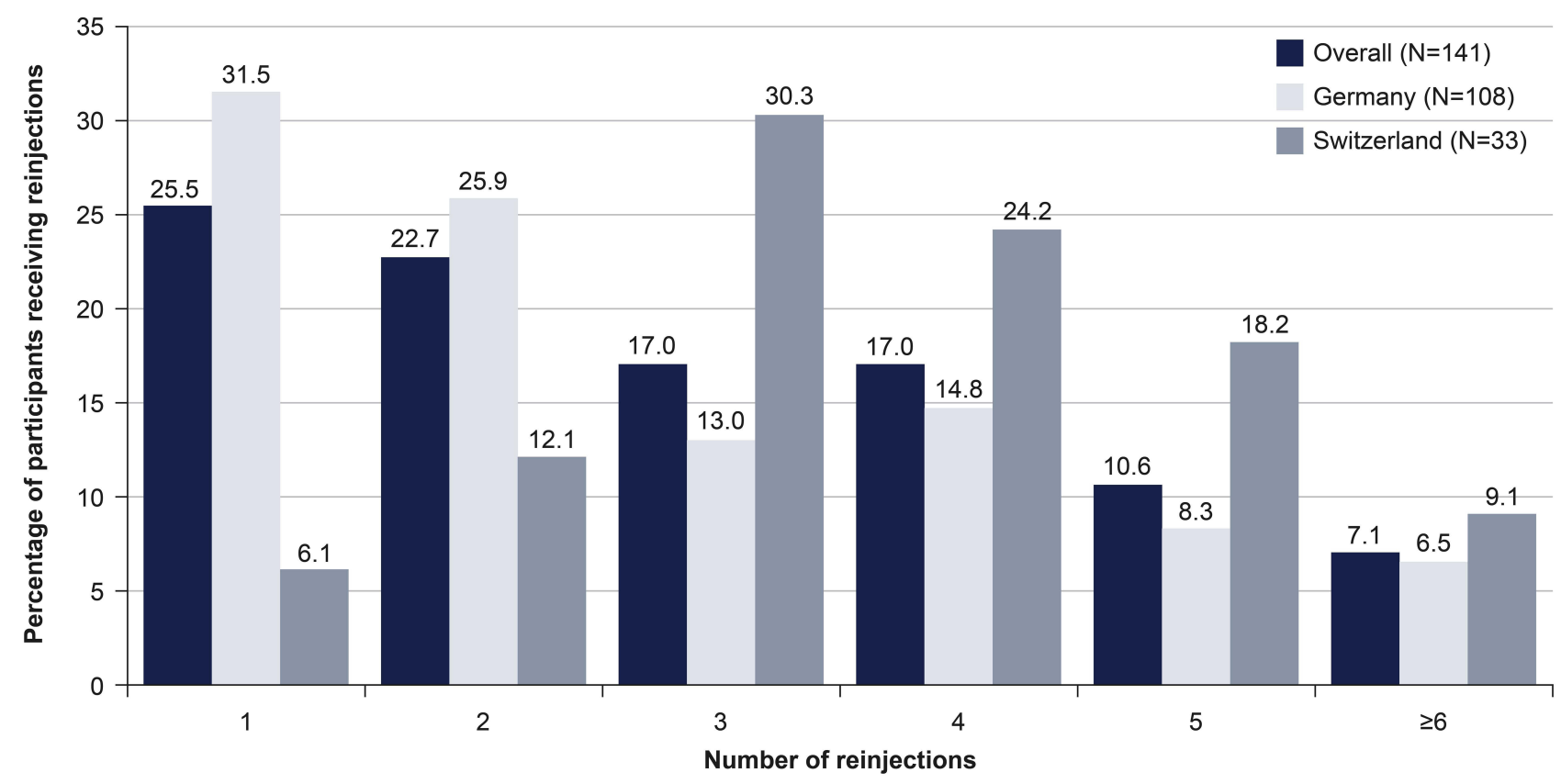

Figure I The graph shows the percentage of participants who received each number of reinjections during the follow-up period for Germany, Switzerland, and overall. 
Table 2 Primary Endpoint: Reinjection Intervals

\begin{tabular}{|c|c|c|c|}
\hline & Germany & Switzerland & Overall \\
\hline $\begin{array}{l}\text { Overall follow-up period } \\
\mathrm{N} \text { (total reinjections) } \\
\text { Mean (SD) (months) } \\
\text { Median (months) }\end{array}$ & $\begin{array}{c}286 \\
6.3(4.6) \\
4.8\end{array}$ & $\begin{array}{c}133 \\
4.6(3.0) \\
3.7\end{array}$ & $\begin{array}{c}419 \\
5.7(4.2) \\
4.6\end{array}$ \\
\hline $\begin{array}{l}\text { Baseline to reinjection I } \\
\mathrm{N} \\
\text { Mean (SD) } \\
\text { Median }\end{array}$ & $\begin{array}{c}108 \\
7.4(5.7) \\
5.5\end{array}$ & $\begin{array}{c}33 \\
5.6(3.3) \\
4.1\end{array}$ & $\begin{array}{c}|4| \\
7.0(5.3) \\
5.1\end{array}$ \\
\hline $\begin{array}{l}\text { Reinjection I to } \\
\text { reinjection } 2 \\
\mathrm{~N} \\
\text { Mean (SD) } \\
\text { Median }\end{array}$ & $\begin{array}{c}74 \\
6.5(4.7) \\
4.9\end{array}$ & $\begin{array}{c}31 \\
5.6(4.6) \\
4.1\end{array}$ & $\begin{array}{c}105 \\
6.2(4.7) \\
4.8\end{array}$ \\
\hline $\begin{array}{l}\text { Reinjection } 2 \text { to } \\
\text { reinjection } 3 \\
\mathrm{~N} \\
\text { Mean (SD) } \\
\text { Median }\end{array}$ & $\begin{array}{c}46 \\
4.9(2.5) \\
4.5\end{array}$ & $\begin{array}{c}27 \\
3.9(1.2) \\
3.9\end{array}$ & $\begin{array}{c}73 \\
4.5(2.1) \\
4.1\end{array}$ \\
\hline $\begin{array}{l}\text { Reinjection } 3 \text { to } \\
\text { reinjection } 4 \\
\mathrm{~N} \\
\text { Mean (SD) } \\
\text { Median }\end{array}$ & $\begin{array}{c}32 \\
4.8(2.3) \\
4.1\end{array}$ & $\begin{array}{c}17 \\
3.9(1.3) \\
3.8\end{array}$ & $\begin{array}{c}49 \\
4.5(2.0) \\
4.1\end{array}$ \\
\hline
\end{tabular}

Abbreviation: SD, standard deviation.

(105.3) and -85.8 (96.1) $\mu \mathrm{m}$ after baseline injection and reinjection 1 , respectively (Table 3 ). Unfortunately, the $\mathrm{n}$ was small for the naïve group for both BCVA $(\mathrm{n}=19,18$, and 13) and CRT (n=21, 10), and a meaningful statistical comparison could not be performed.

\section{Reasons for Reinjection}

There were a total of 419 DEX reinjections. The reasons for reinjections were left to the discretion of the physicians, and the following reasons were collected from the

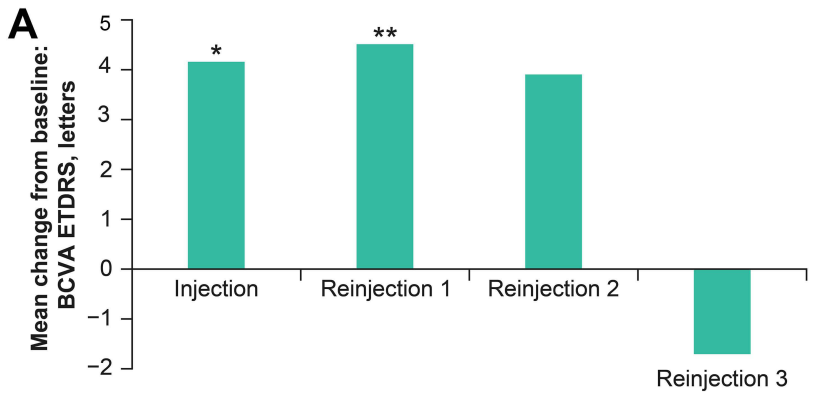

charts: 369 were due to disease progression/relapse (93.77\%), 7 were due to lack of effect of the prior DEX injection (1.9\%), and 16 reported "Other" as the primary reason for reinjection $(4.3 \%)$. The reason for reinjection was missing for 50 reinjections.

\section{Concomitant Treatments}

During follow-up, $34.0 \%(\mathrm{n}=96)$ and $5.7 \%(\mathrm{n}=8)$ of SEs received anti-VEGF treatment and corticosteroid treatment, respectively. The mean (SD) number of anti-VEGF treatments was 3.1 (2.8), and the mean (SD) time from baseline to first anti-VEGF treatment was 9.2 (7.0) months. The mean (SD) number of corticosteroid treatments was $1.3(0.5)$, and the mean (SD) time from baseline to corticosteroid treatment was 13.6 (4.4) months.

Overall, 18 and 19 SEs had focal retinal laser and panretinal photocoagulation treatment during follow-up, respectively. The mean (SD) time from baseline to focal retinal laser and pan-retinal photocoagulation treatment was 7.4 (4.5) and 5.4 (4.5) months, respectively.

\section{Safety}

At baseline, a total of 7 patients had glaucoma and 4 were receiving medication for glaucoma. There were 7 patients who had ocular hypertension at baseline, only two of which were receiving treatment for it. Baseline glaucoma and ocular hypertension data were missing from 2 and 4 patients, respectively. There were 49 phakic patients and 89 pseudophakic patients at baseline; data were missing from 3 patients. Of the phakic patients, 38 had some opacity (nuclear, cortical, posterior subcapsular) at baseline.

Only $14.9 \%$ of patients reported at least $1 \mathrm{AE}$ of special interest in the SE during the study, and a total of 24 AEs of special interest were reported in the SE. The most common were ocular hypertension $(5.7 \%)$, cataract

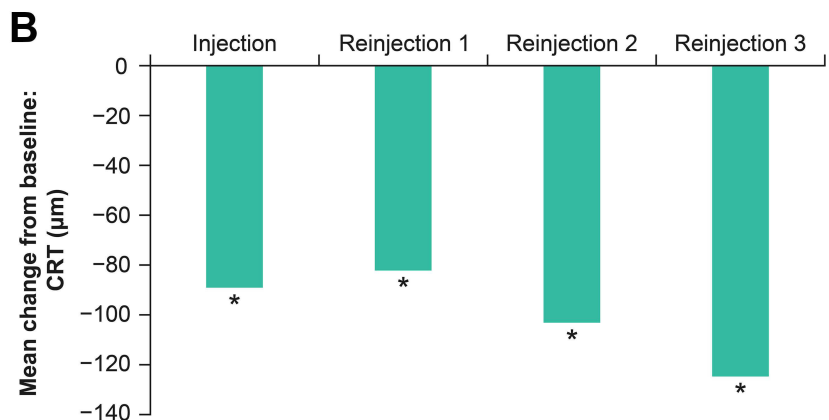

Figure 2 The mean change from baseline 7-12 weeks after each injection for $(\mathbf{A}) B C V A(* P=0.03 ; * * P=0.003)$ and $(\mathbf{B}) C R T(* P \leq 0.00 I)$. Abbreviations: BCVA, best-corrected visual acuity; CRT, central retinal thickness; ETDRS, Early Treatment Diabetic Retinopathy Study. 

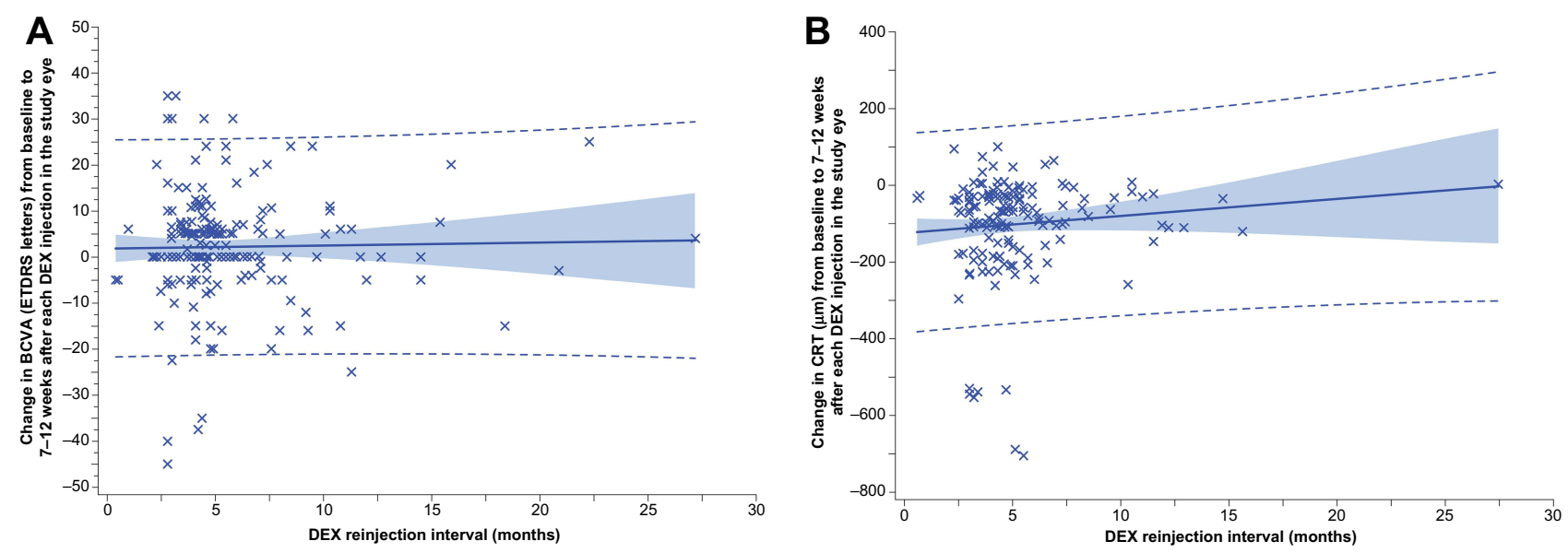

Figure 3 Scatter plots of BCVA and CRT change versus reinjection interval. (A) Change of BCVA (ETDRS letters) 7-I2 weeks after each DEX (re)injection in the SE from baseline. Spearman correlation $=0.03 ; P$-value $=0.66$; (B) Change of CRT $(\mu \mathrm{m}) 7-12$ weeks after each DEX (re)injection in the SE from baseline. Spearman correlation $=0.07$; $P$-value $=0.38$.

Abbreviations: BCVA, best-corrected visual acuity; CRT, central retinal thickness; DEX, dexamethasone intravitreal implant; ETDRS, Early Treatment Diabetic Retinopathy Study; SE, study eye.

or lens opacities $(2.8 \%)$, glaucoma $(2.8 \%)$, ocular bleeding or hemorrhage $(1.4 \%)$, and retinal detachment (tear or hole) (1.4\%). All other AEs occurred in $<1 \%$ of patients. All AEs were considered mild or moderate in severity, with the exception of one AE (ocular bleeding). Table 4 shows the number of patients experiencing $\geq 1 \mathrm{AE}$ and the total number of AEs that occurred between each injection. The greatest percentage AEs of special interest were observed between baseline and the first reinjection.

There does not appear to be a relationship between the number of DEX injections and intraocular pressure (IOP). Ocular hypertension was defined as increased ocular pressure without meeting the criteria for glaucoma, which is nerve damage or vision loss. However, the frequency of reinjections seemed to influence the change in IOP. IOP was higher in patients with a median reinjection interval $\leq 4.6$ months, but an increase was not observable with a median reinjection interval $>4.6$ months. IOP-lowering medication was administered to $17.0 \%$ of patients during the study. The mean (SD) time from baseline to receiving IOP-lowering medication was 6.3 (7.3) months.

Glaucoma was diagnosed in the SE of four additional patients during the study. Two were diagnosed before the first reinjection, one was diagnosed at the time of the first reinjection, and one at the time of the second reinjection. Two patients had glaucoma laser procedures during

Table 3 Secondary Endpoint: Comparison of Naïve and Pretreated Patients in Mean Change from Baseline for BCVA and CRT

\begin{tabular}{|l|c|c|c|c|}
\hline & $\begin{array}{c}\text { BCVA Mean Naïve } \\
\text { at BL, Letters }\end{array}$ & $\begin{array}{c}\text { BCVA Mean Prior Tx } \\
\text { at BL, Letters }\end{array}$ & $\begin{array}{c}\text { CRT Mean Naïve at } \\
\text { BL, } \boldsymbol{\mu m}\end{array}$ & $\begin{array}{c}\text { CRT Mean Prior Tx at } \\
\text { BL, } \boldsymbol{\mu m}\end{array}$ \\
\hline Baseline (SD) & $57.8(16.0)$ & $63.4(14.1)$ & $439.1(143.7)$ & $400.6(119.5)$ \\
\hline $\begin{array}{l}\mathrm{N} \\
\text { Change vs BL 7-12 weeks after } \\
\text { BL (SD) }\end{array}$ & $\begin{array}{c}19 \\
+4.7(11.8)\end{array}$ & $\begin{array}{c}58 \\
+3.0(14.3)\end{array}$ & $-74.3(87.9)$ & $-94.5(105.3)$ \\
\hline $\begin{array}{l}\mathrm{N} \\
\text { Change vs BL 7-12 weeks after } \\
\text { reinjection I (SD) }\end{array}$ & $+4.2(11.1)$ & 53 & $-63.8(106.7)$ & $-85.8(96.1)$ \\
\hline $\begin{array}{l}\text { N } \\
\text { Change vs BL 7-12 weeks after } \\
\text { reinjection 2 (SD) }\end{array}$ & $+1.6(9.6)$ & $+3.5(9.7)$ & - & - \\
\hline
\end{tabular}

Abbreviations: BCVA, best-corrected visual acuity; BL, baseline; CRT, central retinal thickness; SD, standard deviation; Tx, treatment. 
Table 4 Safety Assessment: AEs After Each Injection/Reinjection

\begin{tabular}{|l|c|c|c|}
\hline & Total Patients & Patients Experiencing $\geq$ I AE in SE & Total Number of AEs in SE \\
\hline Between first DEX injection (baseline) and reinjection I & 141 & $9(6.4 \%)$ & II \\
\hline Between first DEX reinjection I and reinjection 2 & 141 & $4(2.8 \%)$ & 4 \\
\hline Between first DEX reinjection 2 and reinjection 3 & 105 & $4(3.8)$ & I \\
\hline Between first DEX reinjection 3 and reinjection 4 & 73 & I (I.4\%) & I \\
\hline Between first DEX reinjection 4 and reinjection 5 & 49 & $1(2.0 \%)$ & I \\
\hline Between first DEX reinjection 5 and reinjection 6 & 25 & I (4.0\%) & \\
\hline
\end{tabular}

Abbreviations: $A E$, adverse event; DEX, dexamethasone intravitreal implant; SE, study eye.

follow-up in the SE, one of which has already been diagnosed with glaucoma at baseline. The mean (SD) time from baseline to the laser procedure was 12.4 (1.2) months.

A total of 4 patients had documented adverse events of cataract or lens opacities in the SE during the study, and three recovered without sequelae. Two incidences were diagnosed prior to the first reinjection, and the other two were diagnosed at the time of the second reinjection. During follow-up, 25 eyes were reported as having changed from phakic to pseudophakic and, of those, 21 had some type of opacity at baseline (data missing from 2 patients). The mean (SD) time from baseline to cataract surgery was $8.1(6.9)$ months.

\section{Discussion}

This study describes DEX injection in the real-world clinical settings of Germany and Switzerland, providing insight into the effectiveness and safety of DEX in realworld practices. The mean number of injections was higher, and the reinjection interval was shorter in Switzerland compared with Germany. The SmPC states that reinjection should be performed after 6 months if the patient experiences disease progression. ${ }^{3}$ While Germany's overall reinjection interval of 6.3 months followed SmPC, Switzerland's reinjection interval of 4.6 months was shorter than recommended. Disease progression was the most common reason for reinjection. Germany's reinjection interval was similar to Italy's (6.7 months) ${ }^{24}$ while Switzerland's was similar to France's (4.9 months). ${ }^{25}$ The differences in reinjection intervals between Switzerland and Germany may be partially due to differences in health insurance and the ease of reimbursement between the two countries. Differences in DME treatment between the two countries create differences in patient selection. In Switzerland, DEX is often the thirdline option after treatment with two other anti-VEGF drugs, while in Germany, it is typically used as the secondline option after the use of another anti-VEGF. This is reflected in the difference between the number of antiVEGF treatment naïve patients in Switzerland (1 [3.03\%]) compared with Germany (44 [40.7\%]). It is also reflected in the mean time between DME diagnosis and baseline; in Switzerland, it was 47.1 months, while in Germany, it was only 23.5 months. This also explains why the number of reinjections is higher in Switzerland. Due to these differences, Swiss patients may be worse responders to anti-VEGF therapies and have a more progressive disease state at baseline, causing the Swiss to have an increased number of reinjections and shorter reinjection intervals.

Overall, there was a trend of improved functional and anatomic effectiveness after DEX injection, although the results showed that there is no relationship between effectiveness and reinjection interval. We only reported details about the first 3 reinjections due to small numbers for the remaining reinjections $(\mathrm{n} \leq 20)$. Although there was no significant correlation, the negative BCVA change after reinjection 3 may be due to the reinjection interval. If BCVA change was separated into groups based on the median reinjection interval, ie, $\leq 4.6$ months or $>4.6$ months, the mean BCVA change from baseline was a positive value, 0.8 , after reinjection 3 in the $\leq 4.6$ months $(\mathrm{n}=23)$ reinjection interval group. Although the number for the $>4$.6-month group was too small $(\mathrm{n}=9)$ to perform statistical comparisons and draw a definitive conclusion, the mean (SD) BCVA change from baseline was -7.1 (13.5) and seemed to be mainly driven by one patient who had a -35 BCVA change from baseline. Due to small numbers, other definitive conclusions, such as the effectiveness of 
DEX between patients who were and were not anti-VEGF treatment naïve, could not be made. Although general trends were observed, the statistical significance could not be determined.

The overall safety profile of DEX was as expected for an ophthalmic steroid treatment or intravitreal injection and was considered good with only $14.9 \%$ of patients presenting with one $\mathrm{AE}$ of special interest in the SE. There was a maximum of 2 AEs per patient, and most were mild or moderate. Incidence of AEs was as expected with an ophthalmic steroid treatment or intravitreal injection. However, the percentage of patients on IOP-lowering medication was lower than expected based on a previous study. ${ }^{9}$ It is difficult to determine if there is a relationship between AEs and the number of injections. However, the results do not indicate it, as the number of AEs did not increase with an increasing number of injections. A relationship may exist between reinjection interval and $\mathrm{AE}$, as IOP increase was greater when the median reinjection interval was $\leq 4.6$ months, but the small sample size of this study prevents a definitive conclusion from being drawn.

The overall effectiveness results were better in the REINFORCE study done in the United States. The mean reinjection interval was 5.0 months, and the mean maximum change in BCVA from baseline was $+9.1,+7.7$, and +7.0 letters after baseline injection, reinjection 1, and reinjection 2, respectively. The mean change in CRT from baseline was $-125.0,-121.2$, and $-140.3 \mu \mathrm{m}$ after baseline injection, reinjection 1 , and 2, respectively. Although this study assessed reinjection interval and effectiveness, the overall mean interval between all DEX injections was determined. In contrast, our study details the mean and median intervals in months between each reinjection. ${ }^{13}$ In a Korean study, the average reinjection interval (with DEX or other anti-VEGF) was 4.4 months, but only $48.9 \%$ were retreated during the 6-month study. Effectiveness was measured at 3 and 6 months, regardless of reinjection. The mean change in BCVA from baseline was approximately +6 and +3 letters, 3 and 6 months after baseline, respectively. The measurements were conducted in $\log$ MAR and converted. Mean change in CRT from baseline was -133.9 and $-79.1 \mu \mathrm{m}, 3$ and 6 months after baseline, respectively. ${ }^{14}$ Both previous studies show that DEX has functional and anatomic outcomes, improving BCVA and CRT in real-world clinical practice and providing support for the findings of this study. The higher effectiveness seen in both studies may be due to the lower baseline BCVA and the higher baseline CRT when compared with this study. In addition, the mean reinjection interval in our study was 5.7 months, which is greater than the other 2 studies. In the REINFORCE study, the percentage of SEs that were phakic was also lower compared with that in this study: $29.4 \%$ and $35.5 \%$, respectively. The Korean study had a much higher percentage of patients who were anti-VEGF treatment naïve (81.7\%) and had a shorter time from DME diagnosis to baseline (17.3 months).

Real-world studies in France ${ }^{15}$ and Spain $^{16}$ showed that DEX improved functional and anatomic outcomes, through improvement in BCVA and central macular thickness (CMT). However, there were only 33 and 84 eyes examined in the studies, respectively. The study conducted in France showed that at peak efficacy, 30\% of eyes achieved a significant change in BCVA ( $>15$ letters) and about $60 \%$ had a CMT of $<300 \mu \mathrm{m} .{ }^{15}$ In the study conducted in Spain, the measurements taken at baseline and during follow-up within 2, 4, and 6 months were obtained. The improvements in both BCVA and CMT were significant upon DEX implant in this study. ${ }^{16}$ A large retrospective study ( $n=340$ eyes), which used the European DME registry, also showed the functional and anatomic efficacy of DEX in a real-world setting. A total of $22.7 \%$ had a $15-$ letter improvement in BCVA and mean (SD) change in CMT was -174 (171) $\mu \mathrm{m} .{ }^{17}$ These three studies also showed greater efficacy in treatment-naïve patients. ${ }^{15-17}$ Another study in Spain $(n=50)$ that only included eyes not previously treated with an intravitreal drug showed that DEX improved BCVA and CMT. The changes from baseline in BCVA and CMT were significant at 2, 4, 6, 12 months, and at the end of the study. ${ }^{12}$ However, none of these studies focused on the reinjection interval or investigated how safety and efficacy could be affected by reinjection interval.

In contrast, results from the Canadian study (CHROME) indicated that the average change in BCVA by Snellen line was not considered significant. However, the study population of patients with DME was small and effectiveness was measured at 6-months, both of which may contribute to the differences seen in this study. In addition, the mean change in CRT from baseline was $-190.9 \mu \mathrm{m}$, which is greater than what was previously observed. $^{11}$

Due to the retrospective and anonymized study design, the assessments were not standardized, and missing data were frequent, both of which are limitations of this study. 
The small sample size was also a limitation, as analysis of certain subgroups could not be performed reasonably. Some results, such as the assessment of effectiveness in naïve versus anti-VEGF-pretreated patients, should be interpreted with caution due to the small numbers. Therefore, it is difficult to draw a meaningful conclusion about effectiveness in naïve anti-VEGF-treated patients.

\section{Conclusions}

This retrospective study provided a detailed report of DEX reinjection intervals in the real-world settings of Germany and Switzerland. Results showed that there does not appear to be a relationship between effectiveness and DEX reinjection interval and there was a good safety profile for DEX. Overall, results indicated that DEX improves BCVA and CRT in real-world clinical practices, consistent with prior studies.

\section{Abbreviations}

AE, adverse event; anti-VEGF, anti-vascular endothelial growth factor; BCVA, best-corrected visual acuity; CMT, central macular thickness; CRT, central retinal thickness; DEX, dexamethasone intravitreal implant; DME diabetic macular edema; IOP, intraocular pressure; SD, standard deviation; SE, study eye; SmPC, summary of product characteristics.

\section{Data Sharing Statement}

This clinical trial data can be requested by any qualified researchers who engage in rigorous, independent scientific research, and will be provided following review and approval of a research proposal and Statistical Analysis Plan and execution of a Data Sharing Agreement. Data requests can be submitted at any time, and the data will be accessible for 12 months, with possible extensions considered. For more information on the process, or to submit a request, visit the following link: https:/www.abbvie.com/our-science/clinicaltrials/clinical-trials-data-and-information-sharing/data-andinformation-sharing-with-qualified-researchers.html.

\section{Acknowledgments}

Writing and editorial assistance was provided to the authors by Stephanie Kuwahara, PhD, of AbbVie.

\section{Funding}

This research was supported by Allergan (prior to its acquisition by AbbVie Inc). Employees of AbbVie participated in the research, interpretation of data, review of the manuscript, and the decision to submit for publication.

\section{Disclosure}

All authors met the ICMJE authorship criteria. Neither honoraria nor payments were made for authorship. Albert J. Augustin has received research support from Bayer, Novartis, Alimera, Allergan, has received compensation for advisory board meetings from Alimera and Allergan, and has received consulting fees from Allergan and Alimera. Matthias D. Becker has received consulting fees from Roche and has stock/patents with Ophthorobotics. Katja Hatz has received research support from Bayer, Novartis, and Allergan, has received compensation for advisory board meetings from Bayer, Novartis, Allergan, and Roche, and has received consulting fees from Kinarus AG. Hakan Kaymak has received research support from Bayer, Novartis, and Allergan and has received compensation for advisory board meetings from Bayer, Novartis, Allergan, and Roche. Andrew Shirlaw is a fulltime employee of AbbVie Inc.

\section{References}

1. Haller JA, Bandello F, Belfort $\mathrm{R}$ Jr, et al. Randomized, sham-controlled trial of dexamethasone intravitreal implant in patients with macular edema due to retinal vein occlusion. Ophthalmology. 2010;117(6):1134-1146.e1133. doi:10.1016/j.ophtha.2010.03.032

2. Chang-Lin J-E, Attar M, Acheampong AA, et al. Pharmacokinetics and pharmacodynamics of a sustained-release dexamethasone intravitreal implant. Invest Ophthalmol Vis Sci. 2011;52(1):80-86. doi:10.1167/iovs.10-5285

3. Allergan. Summary of product characteristics: Ozurdex; 2021. Available from: https://www.ema.europa.eu/en/documents/product-information /ozurdex-epar-product-information_en.pdf. Accessed August 30, 2021.

4. Lee R, Wong TY, Sabanayagam C. Epidemiology of diabetic retinopathy, diabetic macular edema and related vision loss. Eye Vis (Lond). 2015;2:17. doi:10.1186/s40662-015-0026-2

5. Bhagat N, Grigorian RA, Tutela A, Zarbin MA. Diabetic macular edema: pathogenesis and treatment. Surv Ophthalmol. 2009;54 (1):1-32. doi:10.1016/j.survophthal.2008.10.001

6. Funatsu H, Noma H, Mimura T, Eguchi S, Hori S. Association of vitreous inflammatory factors with diabetic macular edema. Ophthalmology. 2009;116(1):73-79. doi:10.1016/j.ophtha.2008.09.037

7. Wang K, Wang Y, Gao L, Li X, Li M, Guo J. Dexamethasone inhibits leukocyte accumulation and vascular permeability in retina of streptozotocin-induced diabetic rats via reducing vascular endothelial growth factor and intercellular adhesion molecule-1 expression. Biol Pharm Bull. 2008;31(8):1541-1546. doi:10.1248/bpb.31.1541

8. Callanan DG, Gupta S, Boyer DS, et al. Dexamethasone intravitreal implant in combination with laser photocoagulation for the treatment of diffuse diabetic macular edema. Ophthalmology. 2013;120 (9):1843-1851. doi:10.1016/j.ophtha.2013.02.018

9. Boyer DS, Yoon YH, Belfort R Jr, et al. Three-year, randomized, sham-controlled trial of dexamethasone intravitreal implant in patients with diabetic macular edema. Ophthalmology. 2014;121 (10):1904-1914. doi:10.1016/j.ophtha.2014.04.024 
10. Boyer DS, Faber D, Gupta S, et al. Dexamethasone intravitreal implant for treatment of diabetic macular edema in vitrectomized patients. Retina. 2011;31(5):915-923. doi:10.1097/IAE.0b013e318206d18c

11. Lam WC, Albiani DA, Yoganathan P, et al. Real-world assessment of intravitreal dexamethasone implant $(0.7 \mathrm{mg})$ in patients with macular edema: the CHROME study. Clin Ophthalmol. 2015;9:1255-1268. doi:10.2147/opth.s80500

12. Menezo M, Roca M, Menezo V, Pascual I. Intravitreal dexamethasone implant Ozurdex in the treatment of diabetic macular edema in patients not previously treated with any intravitreal drug: a prospective 12-month follow-up study. Curr Med Res Opin. 2019;35(12):2111-2116. doi:10.1080/03007995.2019.1652449

13. Singer MA, Dugel PU, Fine HF, Capone A Jr, Maltman J. Real-world assessment of dexamethasone intravitreal implant in DME: findings of the prospective, multicenter REINFORCE study. Ophthalmic Surg Lasers Imaging Retina. 2018;49(6):425-435. doi:10.3928/2325816020180601-07

14. Moon BG, Lee JY, Yu HG, et al. Efficacy and safety of a dexamethasone implant in patients with diabetic macular edema at tertiary centers in Korea. J Ophthalmol. 2016;2016:9810270. doi:10.1155/2016/9810270

15. Majstruk L, Qu-Knafo L, Sarda V, et al. [Dexamethasone intravitreal implant (Ozurdex) in patients with diabetic macular edema: real life safety and efficacy]. J Fr Ophtalmol. 2020;43(3):197-204. French. doi:10.1016/j.jfo.2019.08.010

16. Castro-Navarro V, Cervera-Taulet E, Navarro-Palop C, MonferrerAdsuara C, Hernández-Bel L, Montero-Hernández J. Intravitreal dexamethasone implant Ozurdex ${ }^{\circledR}$ in naïve and refractory patients with different subtypes of diabetic macular edema. BMC Ophthalmol. 2019;19(1):15. doi:10.1186/s12886-018-1022-9

17. Rosenblatt A, Udaondo P, Cunha-Vaz J, et al. A collaborative retrospective study on the efficacy and safety of intravitreal dexamethasone implant (Ozurdex) in patients with diabetic macular edema: the European DME Registry Study. Ophthalmology. 2020;127 (3):377-393. doi:10.1016/j.ophtha.2019.10.005

18. International Society for Pharmacoepidemiology. Guidelines for Good Pharmacoepidemiology Practices (GPP); June, 2015. Available from: https://www.pharmacoepi.org/resources/policies/guidelines-08027/. Accessed August 30, 2021.
19. World Medical Association. World Medical Association Declaration of Helsinki: ethical principles for medical research involving human subjects; 2013. Available from: https://www.wma.net/policies-post /wma-declaration-of-helsinki-ethical-principles-for-medical-researchinvolving-human-subjects/. Accessed August 30, 2021.

20. European Medicines Agency. Guideline on good pharmacovigilance practices (GVP): module VI-Collection, management and submission of reports of suspected adverse reactions to medicinal products (Rev 2). EMA/873138/2011; 2017. Available from: https://www.ema. europa.eu/en/documents/regulatory-procedural-guideline/guidelinegood-pharmacovigilance-practices-gvp-module-vi-collectionmanagement-submission-reports_en.pdf. Accessed August 30, 2021.

21. European Parliament and Council. EU general data protection regulation - regulation (EU) 2016/679; 2016. Available from: http://ec. europa.eu/justice/data-protection/reform/files/regulation_oj_en.pdf. Accessed August 30, 2021.

22. European Network of Centres for Pharmacoepidemiology and Pharmacovigilance. Guide on methodological standards in pharmacoepidemiology (Revision 6). EMA/95098/2010; 2017. Available from: http://www.encepp.eu/standards_and_guidances/documents/ ENCePPGuideofMethStandardsinPE_Rev6.pdf. Accessed August 30, 2021.

23. Gregori NZ, Feuer W, Rosenfeld PJ. Novel method for analyzing Snellen visual acuity measurements. Retina. 2010;30(7):1046-1050. doi:10.1097/IAE.0b013e3181d87e04

24. Mastropasqua R, Toto L, Borrelli E, et al. Morphology and function over a one-year follow up period after intravitreal dexamethasone implant (Ozurdex) in patients with diabetic macular edema. PLoS One. 2015;10(12):e0145663. doi:10.1371/journal.pone.0145663

25. Querques G, Darvizeh F, Querques L, Capuano V, Bandello F, Souied EH. Assessment of the real-life usage of intravitreal dexamethasone implant in the treatment of chronic diabetic macular edema in France. J Ocul Pharmacol Ther. 2016;32(6):383-389. doi:10.1089/jop.2016.0010
Clinical Ophthalmology

\section{Publish your work in this journal}

Clinical Ophthalmology is an international, peer-reviewed journal covering all subspecialties within ophthalmology. Key topics include: Optometry; Visual science; Pharmacology and drug therapy in eye diseases; Basic Sciences; Primary and Secondary eye care; Patient Safety and Quality of Care Improvements. This journal is indexed on PubMed

\section{Dovepress}

Central and CAS, and is the official journal of The Society of Clinical Ophthalmology (SCO). The manuscript management system is completely online and includes a very quick and fair peer-review system, which is all easy to use. Visit http://www.dovepress.com/ testimonials.php to read real quotes from published authors. 